\title{
Developing a comprehensive Cardio-Oncology Program at a Cancer Institute: the Moffitt Cancer Center experience
}

\author{
Michael G. Fradley, ${ }^{1,2}$ Allen C. Brown, ${ }^{2}$ Bernadette Shields, ${ }^{1}$ Federico Viganego,,${ }^{1,2}$ \\ Rongras Damrongwatanasuk, ${ }^{1,2}$ Aarti A. Patel, ${ }^{2}$ Gregory Hartlage, ${ }^{2}$ Natalee Roper, ${ }^{1}$ Julie Jaunese, ${ }^{1}$ \\ Larry Roy, ${ }^{1}$ Roohi Ismail-Khan ${ }^{1,3}$ \\ ${ }^{1}$ Cardio-Oncology Program, H. Lee Moffitt Cancer Center and Research Institute; ${ }^{2}$ Division of Cardiovascular \\ Medicine, University of South Florida; ${ }^{3}$ Women's Oncology Program, H. Lee Moffitt Cancer Center and Research \\ Institute, Tampa, FL, USA
}

\begin{abstract}
Cardio-oncology is a multidisciplinary field focusing on the management and prevention of cardiovascular complications in cancer patients and survivors. While the initial focus of this specialty was on heart failure associated with anthracycline use, novel anticancer agents are increasingly utilized and are associated with many other cardiotoxicities including hypertension, arrhythmias and vascular disease. Since its inception, the field has developed at a rapid pace with the establishment of programs at many major academic institutions and community practices. Given the complexities of this patient population, it is important for providers to possess knowledge of not only cardiovascular disease but also cancer subtypes and their specific therapeutics. Developing a cardio-oncology program at a stand-alone cancer center can present unique opportunities and challenges when compared to those affiliated with other institutions including resource allocation, cardiovascular testing availability and provider education. In this review, we present our experiences establishing the cardio-oncology program at Moffitt Cancer Center and provide guidance to those individuals interested in developing a program at a similar independent cancer institution.
\end{abstract}

Correspondence: Michael G. Fradley, Cardio-Oncology Program, H. Lee Moffitt Cancer Center and Research Institute; and Division of Cardiovascular Medicine, University of South Florida, 2 Tampa General Circle, 5th Floor, Tampa, Florida 33606, USA.

Tel.: +1.813.259.8743. E-mail: mfradley@health.usf.edu

Key words: Cardio-Oncology; cardiotoxicity; chemotherapy.

Conference presentation: data initially presented in abstract form at the Global Cardio-Oncology Summit, October 2015, Nashville, TN, USA.

Received for publication: 17 April 2017.

Revision received: 29 June 2017.

Accepted for publication: 30 June 2017.

This work is licensed under a Creative Commons Attribution NonCommercial 4.0 License (CC BY-NC 4.0).

(C)Copyright M.G. Fradley et al., 2017

Licensee PAGEPress, Italy

Oncology Reviews 2017; 11:340

doi:10.4081/oncol.2017.340

\section{Introduction}

Although cancer and cardiovascular (CV) disease remain the two most common causes of mortality in the United States, survival for both conditions has improved dramatically. The death rate for all cancers declined by 22\% between 1991 and 2011, driven by both improved diagnostic and therapeutic modalities. ${ }^{1}$ Despite these advances, there is increasing recognition that many cancer patients experience CV complications as a result of their therapies. This includes the development of newly diagnosed CV problems, or the exacerbation of previously identified CV disease. Rates of cardiotoxicity from cancer-related therapeutics have been reported to be in excess of $30 \%$, with some events occurring several decades after the completion of treatment. ${ }^{2,3}$ In addition, cardiac toxicity is the second most common cause of morbidity and mortality in cancer survivors. ${ }^{4}$

Oncologists are frequently the first medical providers to observe these cardiotoxicities. Historically these patients would be referred to cardiologists who may not have significant knowledge of the cancer therapeutics and their potential effects on the heart, which has led to variable diagnosis and treatment. ${ }^{5}$ The complexities of treating cancer patients with cardiac complications have led to the development of the new cardiology sub-specialty, cardio-oncology, which is a multidisciplinary field aimed at the prevention and treatment of cardiotoxicities in cancer patients and survivors. ${ }^{6}$ Cardio-oncology clinics are currently expanding at an explosive pace in both academic centers and community practices. 7,8

The comprehensive academic cardio-oncology program at $\mathrm{H}$. Lee Moffitt Cancer Center and Research Institute, in collaboration with the University of South Florida aims to provide cancer patients optimal prevention and treatment of $\mathrm{CV}$ disease using a multi-disciplinary approach. In this article, we discuss the experiences and rationale of setting up a cardio-oncology program at a large cancer institute, emphasizing a combination of patient care (including comprehensive evaluation before, during and/or after cancer therapy), research, as well as education. In addition, we highlight some of the unique opportunities and challenges associated with developing a cardio-oncology program at a cancer-specific hospital.

\section{Cardiotoxicity: opportunities for collaboration}

CV complications of cancer therapy, particularly the development of congestive heart failure (CHF) and cardiomyopathy have been recognized since the 1970 s. $^{9}$ Anthracyclines are a class of 
chemotherapeutics used in the treatment of many different malignancies including breast, sarcoma, leukemia and lymphoma. Rates of heart failure have been reported up to $26 \%$, with higher cumulative dose, female gender, underlying $\mathrm{CV}$ disease and both younger and older age groups increasing this risk. ${ }^{3,10}$ Similarly, trastuzumab, a targeted cancer therapeutic that revolutionized the treatment of certain breast cancers overexpressing the HER2 receptor, has demonstrated rates of CHF between 1-28\%. . $^{3,11,12}$ As such, much of cardio-oncology initially focused on breast cancer patients and those individuals experiencing CHF. Despite this recognition, many patients that have developed cardiomyopathy as a result of these exposures may not receive optimal heart failure (HF) treatments. In one study evaluating patients with an asymptomatic decrease in ejection fraction (EF) after chemotherapy exposure, only $31 \%$ received an ACE inhibitor or angiotensin receptor blocker, while $35 \%$ received a beta blocker and only $42 \%$ were referred for a cardiology consultation. ${ }^{13}$ The use of HF medications is especially important in this population given data demonstrating their early use can result in normalization of EF in almost half of the patients with an anthracycline induced cardiomyopathy. ${ }^{14}$

While HF associated with anthracyclines and anti-HER2 therapies is of significant concern, a variety of other cardiotoxicities are observed with many traditional and novel cancer therapeutics. Therefore, it is essential for cardio-oncologists to possess broad knowledge of these treatments and the associated complications to provide optimal care. Among traditional therapeutics, cardiotoxicity is frequently associated with cisplatin, cyclophosamide, and 5fluorouracil. ${ }^{3,15}$ Cisplatin is associated with arterial thrombosis and both myocardial infarction and stroke in up to $2 \%$ of patients. ${ }^{16}$ Moreover, cisplatin-treated testicular cancer survivors have an increased long-term incidence of coronary artery disease. ${ }^{17,18}$ Cyclophosphamide can be associated with HF, especially with the higher doses used for stem cell transplant preconditioning. ${ }^{19}$ Finally, 5-fluorouracil can lead to myocardial ischemia or infarction, likely due to coronary vasospasm and endothelial dysfunction in up to $10 \%$ of patients. ${ }^{20-22}$

In the last decade, there have been substantial advances in cancer biology leading to many new treatment options including targeted and immune-modulating therapies. These agents are used to treat a variety of malignancies including leukemias, renal cell carcinoma and melanoma, and are associated with many different cardiotoxicities aside from $\mathrm{CHF}$ including arrhythmias, hypertension and vascular disease. For example, tyrosine kinase inhibitors (TKIs) are a class of therapeutics targeting abnormal intracellular signaling pathways in cancer cells. ${ }^{23}$ Unfortunately, they also exhibit off-target effects impacting healthy CV tissues. TKIs targeting the VEGF signaling pathway can lead to significant elevations in blood pressure. ${ }^{24,25}$ Several of the TKIs used to treat chronic myelogenous leukemia are associated with elevated rates of vascular events including strokes and myocardial infarctions. ${ }^{26,27}$ The TKI ibrutinib, used in the treatment of chronic lymphocytic leukemia and mantle cell lymphoma has demonstrated elevated rates of atrial fibrillation and possibly ventricular tachycardia and fibrillation. ${ }^{28-30}$ More recently, immunotherapies including checkpoint-inhibitors are demonstrating significantly improved outcomes in the treatment of many different advanced malignancies. ${ }^{31}$ Despite this, there is now increased recognition of immune-mediated myocarditis, which can lead to rhythm disturbances, HF or death. $^{32}$

It is also important to recognize that cardiovascular complications can be associated with other cancer treatment modalities including radiation therapy. Among patients who receive left sided chest and/or breast radiation or mantle radiation, there is a significantly increased risk of developing accelerated coronary artery dis- ease and valvular dysfunction (especially aortic regurgitation). ${ }^{33,34}$ Patients that receive head and neck radiation are at increased risk for carotid artery disease, stroke as well as autonomic dysfunction due to effects on baroreceptors in the carotid bulb and sinus. ${ }^{35-37}$ In general, these are delayed toxicities, occurring several years to decades after exposure.

With these significant improvements in cancer therapy, there are now over 14 million cancer survivors living in the United States alone. ${ }^{38}$ It is well documented that survivors of many different types of cancer are at increased risk for future $\mathrm{CV}$ events compared to the general population. ${ }^{17,39}$ Rates of $\mathrm{CV}$ disease have been reported between 3-24\% among childhood cancer survivors who have reached the fourth decade of life (age 30-39). ${ }^{40,41}$ Complications of many cancer therapeutics including anthracyclines and radiation may not become apparent for more than 10 years post treatment, and as such, these patients will require long term monitoring and $\mathrm{CV}$ risk reduction by cardio-oncologists.

\section{Developing a Cardio-Oncology Program}

Governing cardiology organizations including the American College of Cardiology (ACC) have recognized the importance of this novel specialty, and have developed a new cardio-oncology members section. A survey in 2014 disseminated to cardiology chairs and program directors about the field of cardio-oncology reported that $27 \%$ of centers have dedicated cardio-oncology programs, with $12 \%$ planning to add these services within 1 year. More than $70 \%$ of respondents agreed that $\mathrm{CV}$ complications are a major issue facing cancer patients, and over half felt that cardiooncology programs improve overall patient care. ${ }^{42}$ Several articles have been published about cardio-oncology program development, however these have focused on programs affiliated with general medical centers rather than those at primary cancer hospitals. ${ }^{7,8,43,44}$

Although cancer centers often have significant resources, allocation for $\mathrm{CV}$ evaluation and testing may not be an obvious priority. Moffitt Cancer Center (MCC), in conjunction with the University of South Florida (USF) developed the comprehensive cardio-oncology program to improve both the quality and outcomes of CV care in cancer patients. The oncology teams recognized the importance of CV services but desired a more collaborative and unified approach which was accomplished through the development of this program, focusing on patient care, education and research (Table 1).

\section{Program leadership}

When creating a new cardio-oncology program, it is essential to identify key individuals who will focus on program development and leadership to ensure the overall success of the program. Given that the primary goal of cardio-oncology is to reduce $\mathrm{CV}$ complications in cancer patients and survivors, the program should be led by a board-certified cardiologist with specific interest and/or training in cardio-oncology, as is the case with our program at MCC. Nevertheless, cardio-oncology is a collaborative, multidisciplinary specialty and it is important to identify an oncologist to serve in a leadership role as well. This individual should have an interest in the cardiotoxicities of cancer therapy and work with the cardiologist on program development, research and education. The oncologist adds legitimacy to the program and demonstrates the commitment and dedication of the program to the cancer patient 
population. In our model at MCC, a cardiologist and breast oncologist work together on all program endeavors with oversight from the chair of cardiovascular sciences (Figure 1).

As with any new program, value to the organization must be demonstrated and this is generally established with support from the senior administration and organizational leadership. Finding an administrative champion for any novel program is essential for both its short- and long-term success. After identifying that individual at MCC, further meetings were conducted to determine the goals and requirements of this program along with the key clinical

Table 1. Key components for a successful comprehensive Cardio-Oncology Program.

\begin{tabular}{ll}
\hline Program Leadership & - Identify both a cardiologist and oncologist to work collaboratively on all aspects of program development \\
Administration & - Demonstrate value of the program to the organizational leadership \\
& - Identify administrator to help with strategic, operational and budgetary planning \\
\hline Outpatient Cardio-Oncology Clinic & - Central location within cancer center, in close proximity to those specialties that refer large numbers of patients \\
& - Ensure adequate space to allow for future expansion and growth \\
& - Ensure appropriate staffing to maximize efficiency \\
& - Coordinate cardio-oncology clinic visits with other cancer center appointments to enhance the patient experience \\
Inpatient Cardio-Oncology Consults & - Inpatient evaluation should be provided by individuals who understand the complexities of cancer patients \\
& - Expeditious and coordinated follow up in the cardio-oncology clinic should be arranged prior to discharge \\
\hline Cardiovascular Testing & - Onsite echocardiography with access to advanced technology including strain and 3D imaging \\
& - Access to electrocardiography in the cardio-oncology clinic \\
& - Incorporation of additional cardiovascular imaging modalities including cardiac MRI \\
& - Formalized transitions for advanced cardiovascular procedures if necessary \\
Cardio-Oncology Nurse Coordinator & - Patient care coordination to minimize treatment disruptions or delays \\
& - Triaging urgent cardiovascular issues \\
& - Patient and staff education \\
& - Staff Education: Attend regular oncology meetings and tumor boards to ensure staff understands the program \\
& and address any questions and concerns \\
\hline Education & - Trainee Education: Integrate dedicated teaching conferences for residents and fellows with a goal to develop \\
& a dedicated cardio-oncology fellowship \\
- Community education: Develop seminars to increase public awareness about cardio-oncology \\
- Offer cardiovascular guidance to oncology clinical trial research staff \\
- Develop dedicated cardio-oncology studies focusing on clinical, translational and basic science research
\end{tabular}

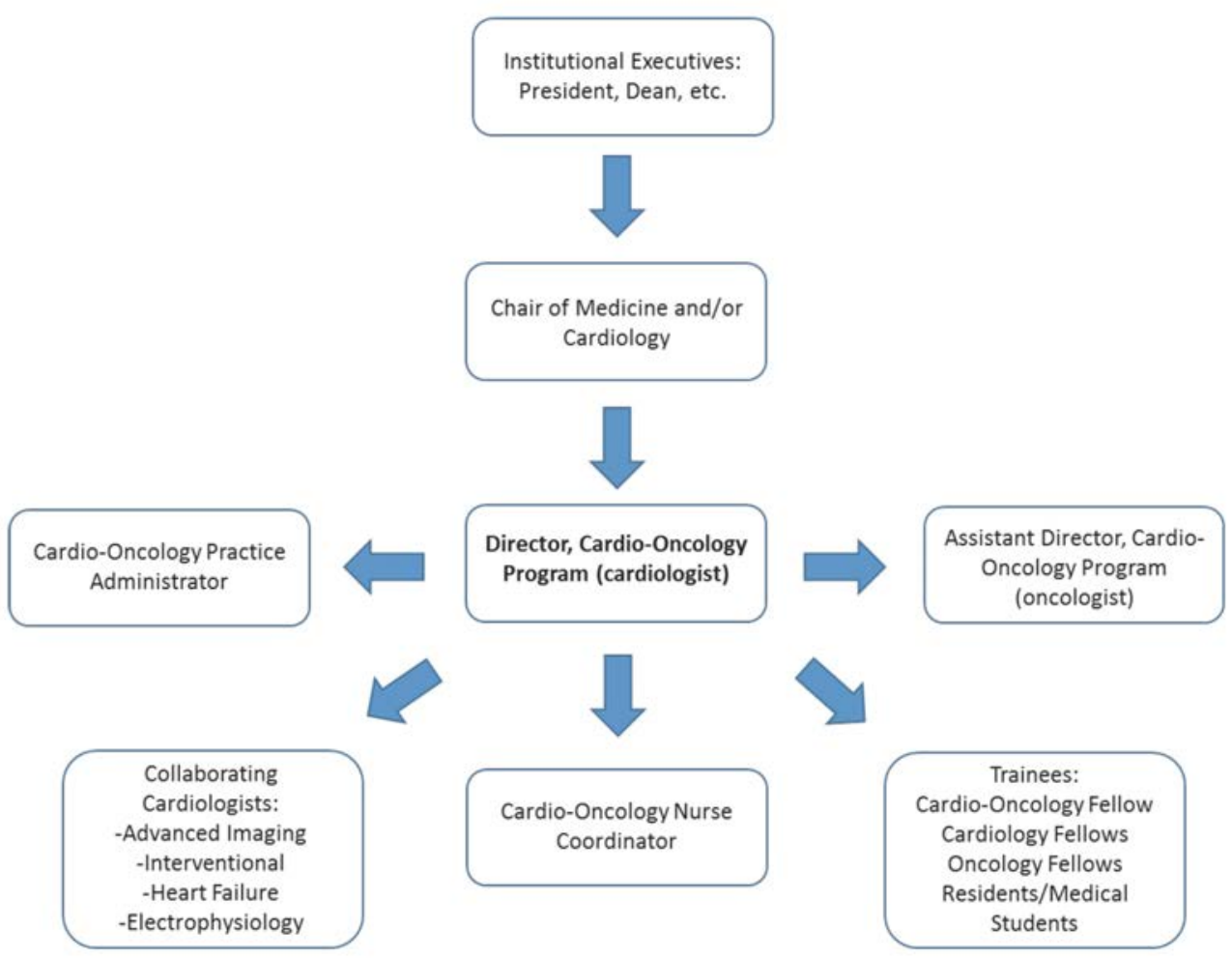

Figure 1. Organization structure of the Moffitt Cancer Center Cardio-Oncology Program. 
and academic needs. We believe it is important to bring a cohesive and well-developed business plan to these administrative meetings as it demonstrates the vision and understanding of the program leadership. After receiving the support from the institution, a dedicated administrator was assigned to the cardio-oncology program to ensure successful operation. The cardio-oncology program administrator is responsible for strategic, operational and budgetary planning, including staff salaries, equipment and supplies, which are all necessary for the overall functioning and success of the program.

\section{Patient care: the outpatient Cardio-Oncology Clinic and Inpatient Consults}

Patient care is the cornerstone of any successful cardio-oncology program. The lack of a dedicated outpatient cardiology clinic at MCC was a clear barrier to effective longitudinal care of patients. As such, a key component of our program was centralizing the cardio-oncology clinic within MCC. A cardio-oncologist working at a cancer specific institution must be prepared to evaluate all types of cardiac patients, including those with $\mathrm{CV}$ issues unrelated to the specific cancer therapies. In addition, there will likely be frequent requests for CV risk assessment before cancer surgeries. While these evaluations may not necessarily represent the true focus of cardio-oncology, they still require a collaborative, multidisciplinary approach to $\mathrm{CV}$ care, which is inherent to the field of cardio-oncology. When evaluating the different referrals to the cardio-oncology clinic in the first 6 months of its existence, comprehensive $\mathrm{CV}$ evaluation prior to starting potentially car- diotoxic treatments (including those agents associated with the development of heart failure, vascular events or arrhythmias) was most common (92, 26\%), followed by arrhythmia/syncope evaluation $(68,19 \%)$ and cardiomyopathy/congestive heart failure evaluation $(48,14 \%)$. The remaining $41 \%(\mathrm{~N}=214)$ were referred for a variety of other issues including preoperative risk assessment or ischemia evaluation (Figure 2). In our clinic, we follow the "ABCDE" approach to risk reduction which has been validated in multiple cancer populations, focusing on blood pressure, lipid and diabetes control, as well as promoting healthy lifestyle choices through education, exercise and nutrition. ${ }^{45}$ Among those patients referred for pre-chemotherapy $\mathrm{CV}$ evaluation, $52 \%(\mathrm{~N}=48)$ were to receive anthracycline-based therapies (ABT) while $48 \%(\mathrm{~N}=44)$ received regimens without anthracyclines (Table 2). For those exposed to ABT, we follow published recommendations for left ventricular ejection fraction assessment (LVEF) at baseline and 612 months after completion of therapy. ${ }^{46} \mathrm{We}$ also incorporate bio-

Table 2. Pre-chemotherapy cardiovascular evaluation ( $\mathrm{N}=92)$.

$\begin{array}{lr}\text { Type of chemotherapy } & \text { N (\%) } \\ \text { Anthracycline based regimens } & 48(52 \%) \\ \text { Non-anthracycline based regimens } & 44(48 \%) \\ \text { HER2 targeted therapies* } & 12(27 \%) \\ \text { Tyrosine kinase inhibitors }^{\circ} & 11(25 \%) \\ \text { Other therapies (including non-HER2 monoclonal antibodies) } & \text { 21 (48\%) }\end{array}$

*Includes: trastuzumab; pertuzumab; 'Includes: axitinib; bosutinib; dabrafenib; imatinib; nilotinib; ponatinib; sunitinib; tremetinib; \#Includes: 5-fluorouracil; abiraterone; bortezomib; carfilzomib; cituximab; fludarabine; gemcitabine; lenalidomide; melphalan; nitrogen mustard; paclitaxel; platinum compounds (carboplatin; cisplatin; oxaliplatin); vincristine.

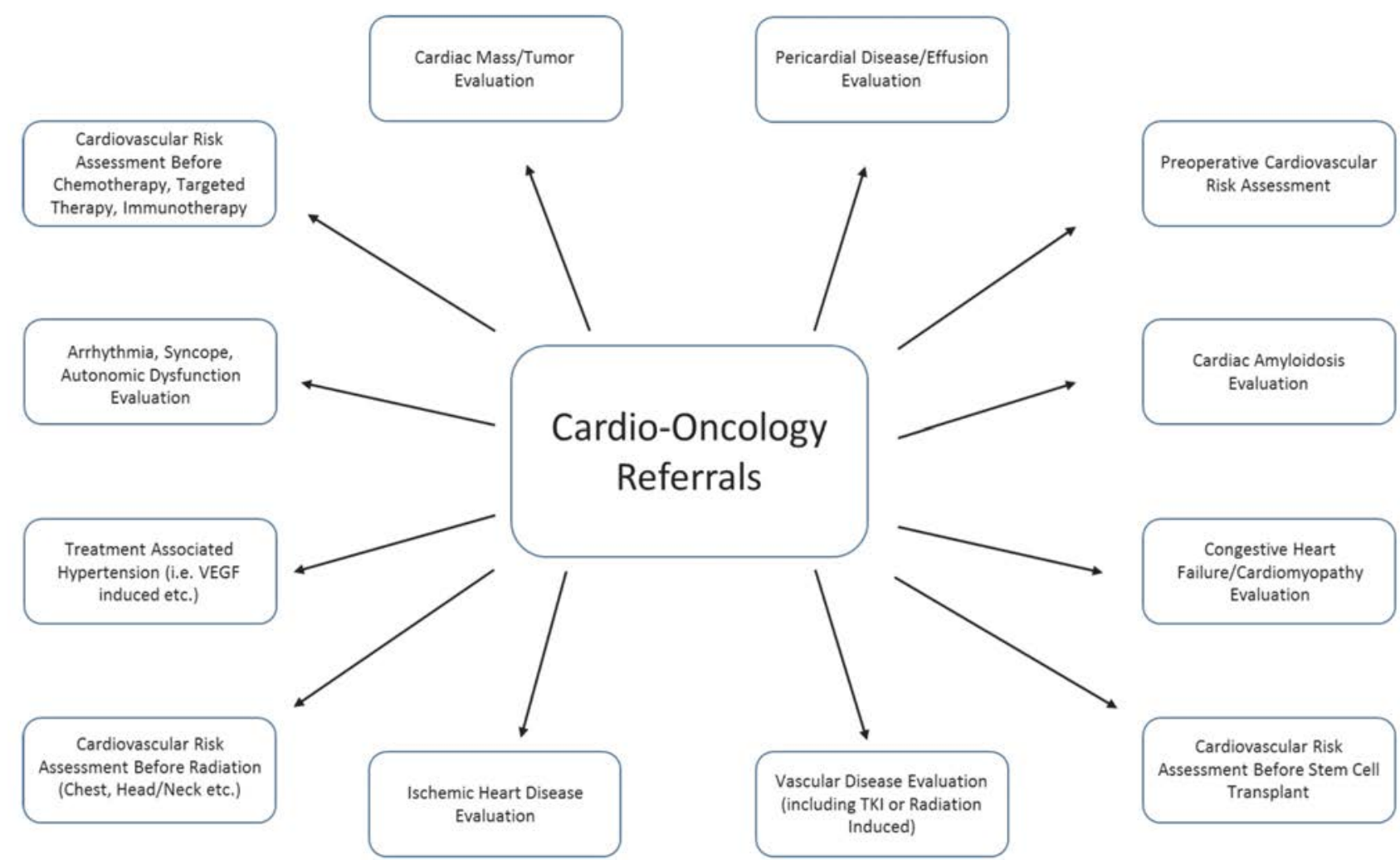

Figure 2. Common Cardio-Oncology referrals at Moffitt Cancer Center. 
marker evaluation with troponin and brain natriuretic peptide (BNP) whenever possible. ${ }^{47,48}$ Among the non-ABT regimens, $52 \%(\mathrm{~N}=23)$ were treated with a TKI and/or HER2 targeted therapy, with $\mathrm{CV}$ recommendations tailored to the specific agent. For example, HER2 targeted agents require regular LVEF assessments whereas TKIs affecting VEGF signaling warrant close BP monitoring. As can be gleaned from these data, the majority of patients referred to a cardio-oncology clinic at a cancer specific institution are seen for monitoring and treatment of $\mathrm{CV}$ complications from cancer therapies, however a significant proportion of patients may also be seen for more general cardiology issues. In order to facilitate additional cardio-oncology referrals, we have started to incorporate $\mathrm{CV}$ recommendations into the MCC clinical pathways, which are treatment and testing algorithms for the various cancers. Moreover, we have created alerts in the electronic order entry sys- tem, reminding providers to consider cardio-oncology evaluation for patients at high risk for $\mathrm{CV}$ complications. The broad range of referrals will serve as the foundation for building a successful cardio-oncology clinic at the cancer center. Consistent with these observations, the clinic at MCC has demonstrated significant growth over the last 2 years (Figure 3). In 2016, 516 new patients were evaluated in the cardio-oncology clinic which is a $52 \%$ increase from the prior year thereby necessitating additional outpatient clinic availability.

Ideally, the cardio-oncology clinic should be located within or near the clinics of those specialties that refer a significant number of patients to the program. At MCC, our clinic space is located near those of the malignant hematology and bone marrow transplant services whom have historically referred the most patients to cardio-oncology (Figure 4). Furthermore, we are adding additional

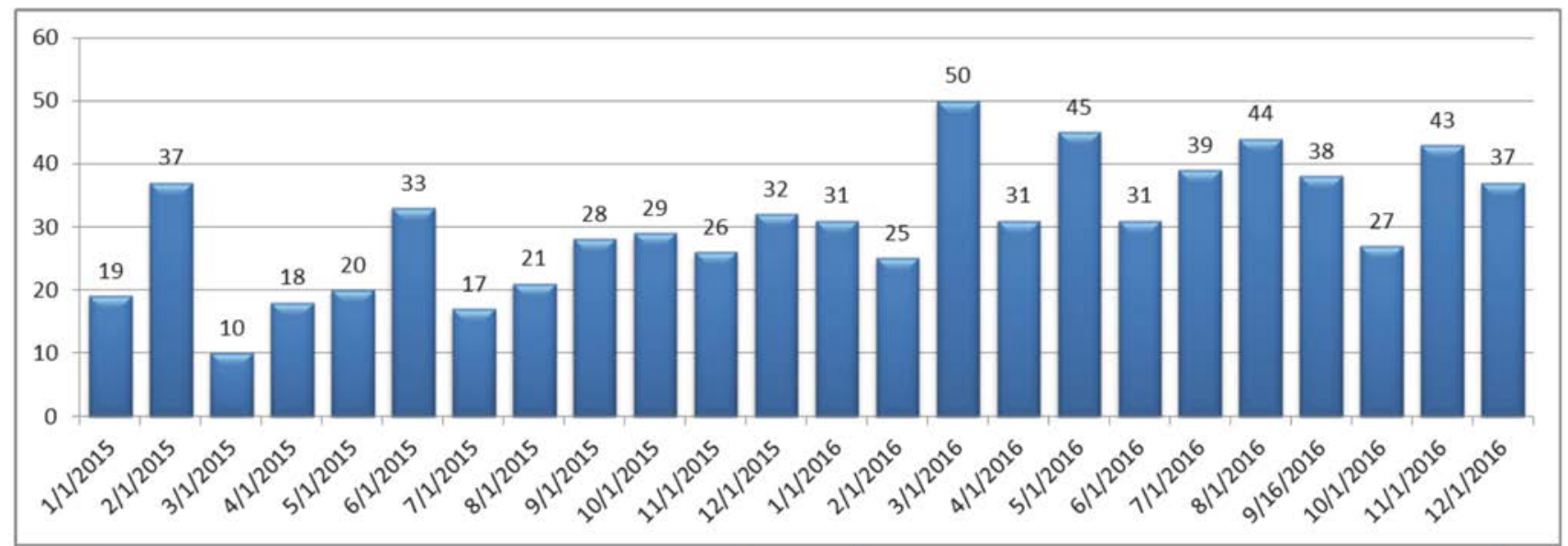

340 total new patients seen in 2015

516 total new patients seen in 2016

\section{$52 \%$ increase year 1 to year 2}

Figure 3. Cardio-Oncology new patient visits in 2015 and 2016.

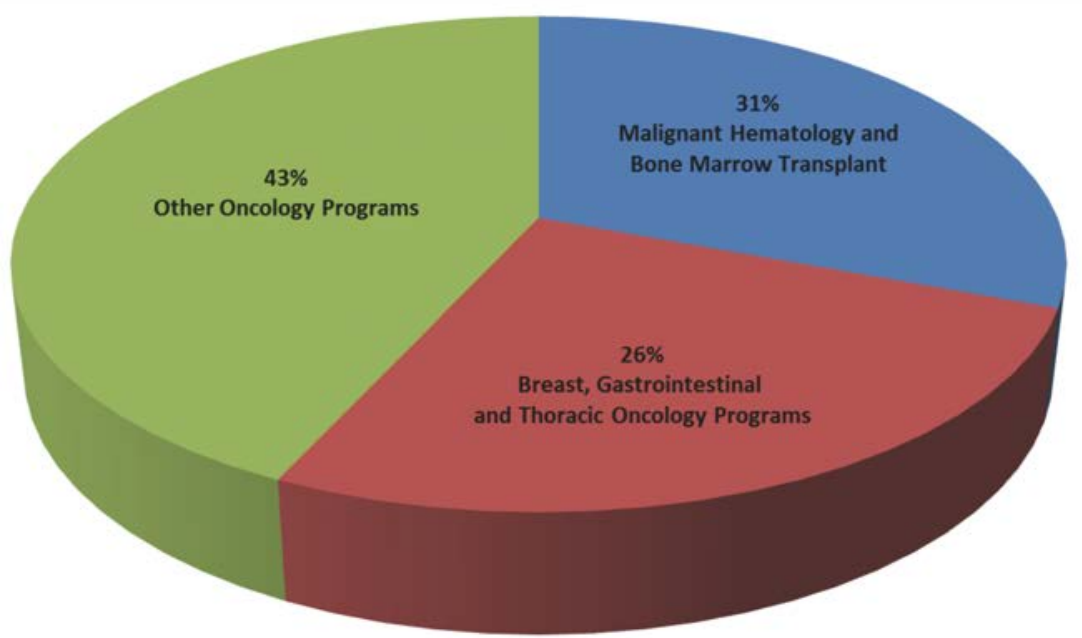

Figure 4. New patient referrals by Oncology Specialty (N=904). 
clinic days to coincide with the increasing volume. We plan to run these concurrently with both the breast and survivorship programs since these populations are also at high risk for CV complications and frequently warrant evaluation.

If the cancer center has inpatient units, a formal consultative service must also be established to provide expeditious evaluation. In our center, we have a cardiology consult service that runs independently of the cardio-oncology clinic. While many consults are for typical CV issues of hospitalized patients such as post-operative atrial fibrillation and chest pain, it is essential that the consultant have more refined knowledge of cardio-oncology as the diagnosis and management of certain $\mathrm{CV}$ conditions are affected by the oncology treatments. Post-discharge follow-up in the cardio-oncology clinic should be coordinated with the patient's upcoming oncology appointments.

\section{Patient care: cardiovascular testing}

Access to $\mathrm{CV}$ testing is an obvious requirement for a functional cardio-oncology program. An electrocardiogram machine should be available in the clinic with staff trained to perform the procedure. We also recommend on-site echocardiography to monitor for cardiac function, which is a necessary requirement those patients receiving HER2 targeted therapies and anthracyclines. While this has been traditionally performed with MUGA (multigated acquisition scan), echocardiography is now the preferred modality for cancer patients. ${ }^{49}$ In addition to 2D echocardiography, advanced procedures such as strain and/or 3D imaging should be incorporated into routine cardio-oncology practice. In our center, we also have access to additional imaging modalities including cardiac MRI, which is especially useful in the non-invasive evaluation of multiple disease states including cardiac amyloidosis and myocarditis, an increasingly recognized complication of checkpoint inhibitors. ${ }^{50,51}$ If not already available, we recommend working with leadership to implement these modalities into the cancer center. Finally a formal relationship should be developed with a general hospital to facilitate seamless transfer of patients for advanced CV procedures including coronary angiography and cardiac device implantation if not performed at the cancer center.

\section{Patient care: Cardio-Oncology Nursing}

Nurses, medical assistants, and advanced practice providers play an important role in the development of a comprehensive cardio-oncology program. At MCC, we created the cardio-oncology nurse coordinator (NC) position, which has been successfully utilized in other $\mathrm{CV}$ and oncology specialties. ${ }^{52,53}$ The $\mathrm{NC}$ is responsible for educating individuals and facilitating awareness throughout the organization about the cardio-oncology program. The NC works with the inpatient and outpatient teams to provide seamless transitions of care. Given the complexities of cancer patients, the $\mathrm{NC}$ ensures that $\mathrm{CV}$ follow-up and testing occur with the least amount of difficulty and disruption to the cancer patient's life. Additional areas of responsibility for the NC include managing patient calls and triaging urgent cardiac issues as well as educating patients about various CV topics including heart failure management, appropriate dietary and lifestyle choices and medication adherence. The NC also plays a vital role in psychosocial assessment, providing resources and referrals to patients that are exhibiting evidence of emotional stress. The NC has been instrumental in improving communication and coordination of care leading to enhanced patient and provider satisfaction with $\mathrm{CV}$ services at MCC.

\section{Cardio-Oncology Program - Education}

Education of clinical staff, trainees and the community is an essential component of a cardio-oncology program. At the beginning stages of development, we recommend presenting at the various oncology service-line meetings, which allows the oncologists to familiarize themselves with the program. After these initial introductions, it is beneficial to regularly attend the different oncology tumor boards. Not only does this enhance visibility and demonstrate commitment of the cardio-oncologist, it is also an opportunity to gain perspective about the oncology treatment plans and potential complications. Additionally, we have instituted cardio-oncology teaching conferences for both cardiology and oncology fellows as well as the internal medicine residents, and have developed community programs to increase public awareness about this topic. These sessions have led to an increased number of patients requesting cardio-oncology evaluation.

Our focus on education has led to the development of a dedicated cardio-oncology fellowship, one of only a few programs in the United States. ${ }^{54}$ This non-accredited fellowship consists of 12 months of clinical exposure, didactic teaching and research. The curriculum is designed so that upon completion of the fellowship, trainees possess expertise in all aspects of cardio-oncology. In addition to regular involvement in the outpatient cardio-oncology clinic and inpatient consultations at MCC, fellows will also have the opportunity to rotate in different oncology clinics such as breast and malignant hematology. If interested, fellows may also participate in the advanced cardiac imaging programs at MCC and USF, gaining exposure to cardiac MRI and CT. Our cardio-oncology fellowship has been quite successful, having trained 2 fellows thus far, with a third fellow recruited for the upcoming academic year.

\section{Cardio-Oncology Program - Research}

Clinical, basic and translational investigation must be incorporated into a cardio-oncology program at a cancer center where clinical trials are being conducted. The cardio-oncologist must provide support to ensure CV safety while preventing unnecessary exclusion. This frequently involves reviewing both echo- and electrocardiograms, with particular attention paid to LV function and QT interval measurements, and may require formal patient consultations. In addition, novel cardio-oncology research is a necessity for any successful program. Though most cardio-oncology projects focus on cardiovascular toxicities and outcomes, a more refined understanding of the cancer-related issues will add additional credibility to the studies. As such, we recommend engaging members of the oncology teams to participate in cardio-oncology research. In our program we are focusing on checkpoint inhibitor myocarditis, and both TKI and proteosome-associated rhythm and vascular abnormalities as these toxicitcies can have significant impact on patient morbidity and mortality.

\section{Cardio-Oncology Program - Future directions}

While tremendous growth and excitement surrounds both our program and field of cardio-oncology as a whole, further scientific evidence documenting improved patient outcomes and standardized practice guidelines are necessary for the future success of the field. As such, we are continuing to expand upon our initial vision and goals for the cardio-oncology program. In the next phase of 
development, we plan to create more formalized protocols for monitoring and treating different cardiotoxicities. Given the data demonstrating the benefits of biomarkers in predicting anthracycline and HER2+ targeted cardiotoxicity, we are creating an algorithm that incorporates routine testing of troponin and BNP at baseline and during treatment based on currently available evidence and recommendations. ${ }^{47,48,55-58}$ These laboratory studies will become a part of a standardized order set to ensure they are routinely assessed at the appropriate times. In addition, we are developing recommended algorithms for the treatment of VEGFinduced hypertension and the prevention of TKI-induced vascular disease, ensuring standardized evaluation and treatment of all patients. ${ }^{26,59}$

We are also developing a formal working relationship with the survivorship program at Moffitt. There is an increasing emphasis on the long-term CV health of cancer survivors and cardio-oncologists must work with survivorship experts to provide collaborative, coordinated care. In addition to geographically localizing our clinic in close proximity to the survivorship clinic, we are actively engaged with the director of the survivorship program to incorporate specific $\mathrm{CV}$ recommendations into the survivorship care plans and facilitate timely evaluations when necessary.

In addition to direct patient care, we are quickly expanding our research endeavors as this is essential for progress in the field. We are routinely engaging coordinators and study monitors of the different oncology trials to ensure patients are evaluated promptly. We believe that this enhanced collaboration will lead to the inclusion of cardio-oncologists in oncology clinical trial design to better evaluate and adjudicate possible CV toxicities. Moreover, we are working to achieve extramural funding for investigator-initiated cardio-oncology studies. We need more thorough research into possible mechanisms of cardiotoxicity as well as preventative interventions as these questions remain incompletely answered. While much of the prior focus has been on anthracyclines and HER2 targeted therapies, we believe that the future of cardiooncology should place more emphasis on the novel therapeutics which are changing the landscape of cancer treatment including TKIs, proteosome inhibitors and immunotherapies. As we focus more on these agents, we will move forward both the scientific and clinical mission of our program and the field as a whole.

\section{Conclusions}

Cardio-oncology is a rapidly developing multidisciplinary field aimed at managing and preventing CV disease in both cancer patients and survivors. Cardio-oncology programs are being established at various academic and non-academic institutions at a rapid pace with interest and support from both the cardiology and oncology communities. Developing a comprehensive cardio-oncology program at a dedicated cancer center presents unique opportunities and challenges when compared to other organizations. Institutional and administrative support is essential for the appropriate allocation of resources and the development of adequate infrastructure to provide comprehensive $\mathrm{CV}$ services at the cancer center. In addition, the foundation for a successful cardio-oncology program is built upon collaboration between cardiologists and oncologists, nursing support to ensure optimal communication and care coordination, as well as research and educational endeavors. Once established, a cardio-oncology program at a cancer hospital can ensure patients receive optimal and collaborative $\mathrm{CV}$ and oncology care leading to improved patient and provider satisfaction, and ultimately better treatment outcomes.

\section{References}

1. Siegel RL, Miller KD, Jemal A. Cancer statistics, 2015. CA Cancer J Clin 2015;65:5-29.

2. Bovelli D, Plataniotis G, Roila F, Group EGW. Cardiotoxicity of chemotherapeutic agents and radiotherapy-related heart disease: ESMO Clinical Practice Guidelines. Ann Oncol 2010;21:v277-82.

3. Yeh ET, Bickford CL. Cardiovascular complications of cancer therapy: incidence, pathogenesis, diagnosis, and management. J Am Coll Cardiol 2009;53:2231-47.

4. Daher IN, Daigle TR, Bhatia N, Durand JB. The prevention of cardiovascular disease in cancer survivors. Tex Heart Inst $\mathrm{J}$ 2012;39:190-8.

5. Chen CL, Steingart R. Cardiac disease and heart failure in cancer patients: is our training adequate to provide optimal care? Heart Fail Clin 2011;7:357-62.

6. Albini A, Pennesi G, Donatelli F, et al. Cardiotoxicity of anticancer drugs: the need for cardio-oncology and cardio-oncological prevention. J Natl Cancer Inst 2010;102:14-25.

7. Sulpher J, Mathur S, Graham N, et al. Clinical experience of patients referred to a multidisciplinary cardiac oncology clinic: an observational study. J Oncol 2015;2015:671232.

8. Okwuosa TM, Akhter N, Williams KA, DeCara JM. Building a cardio-oncology program in a small- to medium-sized, nonprimary cancer center, academic hospital in the USA: challenges and pitfalls. Future Cardiol 2015;11:413-20.

9. Von Hoff DD, Rozencweig M, Layard M, et al. Daunomycininduced cardiotoxicity in children and adults. A review of 110 cases. Am J Med 1977;62:200-8.

10. Swain SM, Whaley FS, Ewer MS. Congestive heart failure in patients treated with doxorubicin: a retrospective analysis of three trials. Cancer 2003;97:2869-79.

11. Suter TM, Procter M, van Veldhuisen DJ, et al. Trastuzumabassociated cardiac adverse effects in the herceptin adjuvant trial. J Clin Oncol 2007;25:3859-65.

12. Guarneri V, Lenihan DJ, Valero V, et al. Long-term cardiac tolerability of trastuzumab in metastatic breast cancer: the M.D. Anderson Cancer Center experience. J Clin Oncol 2006;24:4107-15.

13. Yoon GJ, Telli ML, Kao DP, et al. Left ventricular dysfunction in patients receiving cardiotoxic cancer therapies are clinicians responding optimally? J Am Coll Cardiol 2010;56:1644-50.

14. Cardinale D, Colombo A, Lamantia G, et al. Anthracyclineinduced cardiomyopathy: clinical relevance and response to pharmacologic therapy. J Am Coll Cardiol 2010;55:213-20.

15. Zamorano JL, Lancellotti P, Rodriguez Munoz D, et al. 2016 ESC Position Paper on cancer treatments and cardiovascular toxicity developed under the auspices of the ESC Committee for Practice Guidelines: The Task Force for cancer treatments and cardiovascular toxicity of the European Society of Cardiology (ESC). Eur Heart J 2016;37:2768-801.

16. Moore RA, Adel N, Riedel E, et al. High incidence of thromboembolic events in patients treated with cisplatin-based chemotherapy: a large retrospective analysis. J Clin Oncol 2011;29:3466-73.

17. Haugnes HS, Wethal T, Aass N, et al. Cardiovascular risk factors and morbidity in long-term survivors of testicular cancer: a 20-year follow-up study. J Clin Oncol 2010;28:4649-57.

18. Huddart RA, Norman A, Shahidi M, et al. Cardiovascular disease as a long-term complication of treatment for testicular cancer. J Clin Oncol 2003;21:1513-23.

19. Braverman AC, Antin JH, Plappert MT, et al. Cyclophosphamide cardiotoxicity in bone marrow transplanta- 
tion: a prospective evaluation of new dosing regimens. J Clin Oncol 1991;9:1215-23.

20. Polk A, Vistisen K, Vaage-Nilsen M, Nielsen DL. A systematic review of the pathophysiology of 5-fluorouracil-induced cardiotoxicity. BMC Pharmacol Toxicol 2014;15:47.

21. Kosmas C, Kallistratos MS, Kopterides P, et al. Cardiotoxicity of fluoropyrimidines in different schedules of administration: a prospective study. J Cancer Res Clin Oncol 2008;134:75-82.

22. Frickhofen N, Beck FJ, Jung B, et al. Capecitabine can induce acute coronary syndrome similar to 5-fluorouracil. Ann Oncol 2002;13:797-801.

23. Krause DS, Van Etten RA. Tyrosine kinases as targets for cancer therapy. N Engl J Med 2005;353:172-87.

24. Nazer B, Humphreys BD, Moslehi J. Effects of novel angiogenesis inhibitors for the treatment of cancer on the cardiovascular system: focus on hypertension. Circulation 2011;124:1687-91.

25. Hall PS, Harshman LC, Srinivas S, Witteles RM. The frequency and severity of cardiovascular toxicity from targeted therapy in advanced renal cell carcinoma patients. JACC Heart Fail 2013;1:72-8.

26. Moslehi JJ, Deininger M. Tyrosine kinase inhibitor-associated cardiovascular toxicity in chronic myeloid leukemia. J Clin Oncol 2015;33:4210-18.

27. Li W, Croce K, Steensma DP, et al. Vascular and metabolic implications of novel targeted cancer therapies: focus on kinase inhibitors. J Am Coll Cardiol 2015;66:1160-78.

28. Byrd JC, Brown JR, O'Brien S, et al. Ibrutinib versus ofatumumab in previously treated chronic lymphoid leukemia. $\mathrm{N}$ Engl J Med 2014;371:213-23.

29. Yun S, Vincelette ND, Acharya U, Abraham I. Risk of atrial fibrillation and bleeding diathesis associated with ibrutinib treatment: a systematic review and pooled analysis of four randomized controlled trials. Clin Lymphoma Myeloma Leuk 2017; 17:31-7e13.

30. Lampson BL, Yu L, Glynn RJ, et al. Ventricular arrhythmias and sudden death in patients taking ibrutinib. Blood 2017 [Epub ahead of print].

31. Jain V, Bahia J, Mohebtash M, Barac A. Cardiovascular complications associated with novel cancer immunotherapies. Curr Treat Options Cardiovasc Med 2017;19:36.

32. Johnson DB, Balko JM, Compton ML, et al. Fulminant myocarditis with combination immune checkpoint blockade. $\mathrm{N}$ Engl J Med 2016;375:1749-55.

33. Darby SC, Ewertz M, McGale P, et al. Risk of ischemic heart disease in women after radiotherapy for breast cancer. N Engl J Med 2013;368:987-98.

34. Bijl JM, Roos MM, van Leeuwen-Segarceanu EM, et al. Assessment of valvular disorders in survivors of Hodgkin's lymphoma treated by mediastinal radiotherapy +/- chemotherapy. Am J Cardiol 2016;117:691-6.

35. Huang TL, Hsu HC, Chen HC, et al. Long-term effects on carotid intima-media thickness after radiotherapy in patients with nasopharyngeal carcinoma. Radiat Oncol 2013;8:261.

36. Halak M, Fajer S, Ben-Meir H, et al. Neck irradiation: a risk factor for occlusive carotid artery disease. Eur J Vasc Endovasc Surg 2002;23:299-302.

37. Huang CC, Huang TL, Hsu HC, et al. Long-term effects of neck irradiation on cardiovascular autonomic function: a study in nasopharyngeal carcinoma patients after radiotherapy. Muscle Nerve 2013;47:344-50.

38. DeSantis CE, Lin CC, Mariotto AB, et al. Cancer treatment and survivorship statistics, 2014. CA Cancer J Clin 2014;64:252-71.
39. Chow EJ, Mueller BA, Baker KS, et al. Cardiovascular hospitalizations and mortality among recipients of hematopoietic stem cell transplantation. Ann Intern Med 2011;155:21-32.

40. Mulrooney DA, Armstrong GT, Huang S, et al. Cardiac outcomes in adult survivors of childhood cancer exposed to cardiotoxic therapy: a cross-sectional study. Ann Intern Med 2016;164:93-101.

41. Armstrong GT, Chen Y, Yasui Y, et al. Reduction in late mortality among 5-year survivors of childhood cancer. N Engl J Med 2016 [Epub ahead of print].

42. Barac A, Murtagh G, Carver JR, et al. Cardiovascular health of patients with cancer and cancer survivors: a roadmap to the next level. J Am Coll Cardiol 2015;65:2739-46.

43. Barros-Gomes S, Herrmann J, Mulvagh SL, et al. Rationale for setting up a cardio-oncology unit: our experience at Mayo Clinic. Cardio-Oncology 2016;2(5).

44. Snipelisky D, Park JY, Lerman A, et al. How to develop a cardio-oncology clinic. Heart Fail Clin 2017;13:347-59.

45. Montazeri K, Unitt C, Foody JM, et al. ABCDE steps to prevent heart disease in breast cancer survivors. Circulation 2014;130:e157-9.

46. Armenian SH, Lacchetti C, Barac A, et al. Prevention and monitoring of cardiac dysfunction in survivors of adult cancers: american society of clinical oncology clinical practice guideline. J Clin Oncol 2017;35:893-911.

47. Ky B, Putt M, Sawaya H, et al. Early increases in multiple biomarkers predict subsequent cardiotoxicity in patients with breast cancer treated with doxorubicin, taxanes, and trastuzumab. J Am Coll Cardiol 2014;63:809-16.

48. Cardinale D, Sandri MT, Martinoni A, et al. Left ventricular dysfunction predicted by early troponin I release after highdose chemotherapy. J Am Coll Cardiol 2000;36:517-22.

49. Plana JC, Galderisi M, Barac A, et al. Expert consensus for multimodality imaging evaluation of adult patients during and after cancer therapy: a report from the American Society of Echocardiography and the European Association of Cardiovascular Imaging. J Am Soc Echocardiogr 2014;27:91139.

50. De Smet K, Verdries D, Tanaka K, et al. MRI in the assessment of non ischemic myocardial diseases. Eur J Radiol 2012;81:1546-8

51. Moslehi JJ, Johnson DB, Sosman JA. Myocarditis with immune checkpoint blockade. N Engl J Med 2017;376:292.

52. Sillman C, Morin J, Thomet C, et al. Adult congenital heart disease nurse coordination: Essential skills and role in optimizing team-based care a position statement from the International Society for Adult Congenital Heart Disease (ISACHD). Int J Cardiol 2017;229:125-31.

53. Johnson SA, Giesie PD, Ireland AM, et al. On the scene: developing a nurse care coordinator role at city of hope. Nurs Adm Q 2016;40:39-50.

54. Lenihan DJ, Hartlage G, DeCara J, et al. Cardio-oncology training: a proposal from the International Cardioncology Society and Canadian Cardiac Oncology Network for a new multidisciplinary specialty. J Card Fail 2016;22:465-71.

55. Cardinale D, Sandri MT, Colombo A, et al. Prognostic value of troponin I in cardiac risk stratification of cancer patients undergoing high-dose chemotherapy. Circulation 2004;109:274954.

56. Cardinale D, Colombo A, Torrisi R, et al. Trastuzumabinduced cardiotoxicity: clinical and prognostic implications of troponin I evaluation. J Clin Oncol 2010;28:3910-6.

57. Skovgaard D, Hasbak P, Kjaer A. BNP predicts chemotherapyrelated cardiotoxicity and death: comparison with gated equi- 
librium radionuclide ventriculography. PLoS One 2014;9:e96736.

58. Zardavas D, Suter TM, Van Veldhuisen DJ, et al. Role of troponins I and $\mathrm{T}$ and $\mathrm{N}$-terminal prohormone of brain natriuretic peptide in monitoring cardiac safety of patients with earlystage human epidermal growth factor receptor 2-positive breast cancer receiving trastuzumab: a herceptin adjuvant study cardiac marker substudy. J Clin Oncol 2017;35:878-84.

59. Brinda BJ, Viganego F, Vo T, et al. Anti-VEGF-induced hypertension: a review of pathophysiology and treatment options. Curr Treat Options Cardiovasc Med 2016;18:33. 